We describe a simple and effective IVT technique which reduces severity and impact of these problems, and could be adopted as the default technique. It improves control of the injection process offering additional risk reduction in patients with the aforementioned difficulties. The technique has been adopted in real injection clinics. A series of photographs (Fig. 1a-f) were taken using a volunteer (Mary Awad) for demonstration of the technique without delivering an IVT, illustrating the advantages in a superotemporal IVT.

The injector places a Barraquer speculum with solid flat blade (Fig. 1a). Figure 1a further shows inferonasal gaze without squeezing, demonstrating a large area of superotemporal sclera for injection. Figure $1 \mathrm{~b}$ illustrates inferonasal gaze, with purposeful hard eye squeezing. The PA is significantly narrowed, limiting available injection sites. Figure 1c was taken after eliciting a sudden severe squeeze (with upward globe movement) secondary to unexpected instillation of G. Proxymetacaine onto the ocular surface and exposed eyelashes by the injector in an attempt to alleviate the problem.

Figure 1d illustrates hand positioning for the modified IVT technique. The injector instructs the patient to look inferonasally, holding the syringe in their non-dominant hand between thumb and index finger. The point of injection is marked superotemporally with a calliper. The ring finger is placed on the upper solid part of the speculum and firm downward pressure applied, stabilising the globe in its downward gaze position, offering increased resistance to sudden ocular movements, securing the globe in a more stable position. The authors find it reduced narrowing of the PA during squeezing, maintaining a more constant distance between the upper speculum/eyelid margin and injection site. The dominant hand is free to inject the drug.

This technique offers significant advantages over alternative methods of globe stabilisation, including utilisation of forceps, which results in a one-handed injection technique. A right-handed injector, if desired, can use this technique with their nondominant hand to stabilise the speculum either standing superiorly for left eyes or indeed standing on the right side of the patient for right eye injections, making the technique easy to learn and perform.

Acknowledgements We would like to acknowledge Andrew Martin from the photography department at the Royal Derby Hospital for taking time to acquire the images.

\section{Compliance with ethical standards}

Conflict of interest The authors declare that they have no conflict of interest.

\title{
Do patients only remember who performed their cataract surgery if complications occur?
}

\author{
Safa Elhassan ${ }^{1} \cdot$ Gwyn Samuel Williams ${ }^{1}$
}

Received: 12 February 2018 / Accepted: 18 February 2018 / Published online: 27 March 2018

(c) The Royal College of Ophthalmologists 2018

\section{Introduction}

It is well recognised that patients have poor memory of clinical encounters and are surprisingly bad at recalling

Gwyn Samuel Williams

gwynwilliams@doctors.org.uk

1 Department of Ophthalmology Singleton Hospital Sketty Lane Swansea Wales SA2 8QA UK information discussed during consultations [1]. It has been variously hypothesised that patient literacy levels play an important role in patient recall of clinical information, and there have been suggestions that leaflets and other means of conveying information may be beneficial in aiding patient understanding [2]. With regard to cataract surgery, this has been extended to advocating a multimedia-assisted informed consent process, even in older patients, in order to assist patient recall and understanding of all the risks and benefits. 
There is a saying among cataract surgeons in Wales that patients only ever remember their surgeon if they suffered a complication. To our knowledge, there is no study that has officially examined this issue, and in light of the poor memory of patients in general and patients undergoing cataract surgery in particular we aimed to assess whether there was truth in this old Welsh adage [3].

\section{Methods}

Post-operative cataract extraction and intraocular lens implant patients seen in outpatient ophthalmology clinics or the Day Surgery Unit at Singleton Hospital, Swansea, were asked whether they recalled the name of the surgeon who performed their cataract operation. They were then asked whether the operation had gone well and their result compared with the information contained within the clinical record. Patients were included if they had undergone cataract surgery within the preceding 3 months and if they had both eyes operated on within this period, only the most recent operation was included in this study. If the patient had been operated on by a registrar or specialty doctor but named their responsible consultant this was determined to be positive recall. If the patient determined a complication had taken place, even though the operation note did not indicate that this was the case, the patient was included in the complication group. This study was conducted from November 2016 to January 2018.

\section{Results}

In total, 409 patients were included in this study, of whom 29 claimed to have suffered a 'complication'. There were 13 true intraoperative complications in this group, with the remaining 16 patients suffering from conditions such as post-operative uveitis or a dry eye after the procedure. True complications included posterior capsule rupture, dropped nucleus, anterior capsule tear and zonular dehiscence.

Of the 380 patients who had not suffered a perceived complication, 61 recalled the identity of their surgeon (16.1\%). Of the 29 patients who had suffered a perceived complication, 5 patients recalled the identity of their surgeon $(17.2 \%)$. Chi-square analysis of these figures did not reveal a statistically significant difference between the two groups.

\section{Discussion}

In the brave new world of informed consent, much emphasis is placed on involving the patient in the process and on weighing up the best means of conveying information, so that it can be recalled and used in a meaningful manner for a valid consenting process to take place. Perhaps, it is taken for granted that if a patient is expected to remember complicated information about the operation they are to undergo, they will also remember the name of their surgeon. An extrapolation of this, and perhaps the origin of the saying that 'patients remember bad surgeons', is that the more eventful the procedure, the more memorable the surgeon. This is the first study to demonstrate that not only do patients not in fact remember their surgeon's name with greater clarity if the procedure is not perceived to have gone as planned, but they do not tend to remember their names very much at all.

In both groups, less than a fifth of patients successfully recalled the name of their surgeon. This information can either suggest that yet more information may need to be provided to the patient, including perhaps the name of their surgeon and their complication rate; or indeed that the studies which demonstrate abysmal patient recall of important clinical facts coupled with this study demonstrate that the entire process of a valid clinical consent needs to be rethought. It may be cold comfort to the surgeon suffering an intraoperative complication that the patient will not recall their name, if the consenting process leading up to that point may not be entirely valid.

\section{Compliance with ethical standards}

Conflict of interest The authors declare that they have no conflict of interest.

\section{References}

1. Kessels RPC. Patients' memory for medical information. J R Soc Med. 2003;96:219-22.

2. Safeer RS, Keenan J. Health literacy: the gap between physicians and patients. Am Fam Physician. 2005;72:463-8.

3. Tipotsch-Maca SM, Varsits RM, Ginzel C, Vecsei-Marlovitz PV. Effect of multimedia-assisted informed consent procedure on the information gain, satisfaction, and anxiety of cataract surgery patients. J Cataract Refract Surg. 2016;42:110-6. 